\title{
Calculation and verification of formula for the range of sprinklers based on jet breakup length
}

\author{
Yue Jiang, Hong Li*, Chao Chen, Qingjiang Xiang \\ (Research Center of Fluid Machinery Engineering and Technology, Jiangsu University, Zhenjiang 212013, China)
}

\begin{abstract}
Jet breakup length is an important parameter which reflects the length of sprinkler range. Based on the linear instability theory, the dispersion equation of cylindrical jet was established and the theoretical value of jet breakup length was calculated. The jet breakup length and initial amplitude of surface wave were measured by applying the high-speed photography technology. Meanwhile, the numerical simulation was conducted by combining Level Set-VOF method for describing the jet breakup length to verify the theoretical and experimental results. Within the jet velocity and working pressure range of discussion, the results of comparison showed that the theoretical analysis gave a reasonable explanation to the influence of jet velocity, nozzle diameter and nozzle cone angle on jet breakup length. Comparing the theoretical value of jet breakup length with the experimental and simulated values, the three results accorded one another. The experimental jet breakup lengths were the lowest and the simulation values were the largest, and the relative error was less than $10 \%$, especially the theoretical value was closer to the average value. For choosing the theoretical calculation of jet breakup length, a semi-empirical and semi-theoretical formula of range for the rotating sprinkler was concluded by the curve fitting method and the fitting formula was verified. The results showed the high accuracy of the ranges determined by this formula and the average relative error was less than $2.5 \%$. The new formula was in good agreement with the data of different types of sprinklers comparing with other empirical formulas, and the error was only $5 \%$. Meanwhile, the possibility of using this formula widely to determine the ranges of same series of sprinkler was confirmed.
\end{abstract}

Keywords: sprinkler, irrigation, formula, cylindrical jet, jet breakup length, dispersion equation, high speed photography, Level Set-VOF method

DOI: $10.25165 /$ j.ijabe.20181101.2777

Citation: Jiang Y, Li H, Chen C, Xiang Q J. Calculation and verification of formula for the range of sprinklers based on jet breakup length. Int J Agric \& Biol Eng, 2018; 11(1): 49-57.

\section{Introduction}

Sprinklers are the key component of sprinkling irrigation system, among which rotary sprinkler is being used by more people in the world. It is an initial spray produced by injecting a water jet onto an orthogonal deflector, resulting in thin, unstable, radially expanding streams. The quality of sprinkler irrigation will be directly affected by the hydraulic performance and performance characteristic of sprinklers. Sprinkler range is the main performance index which determines the size of wetted area and irrigation intensity ${ }^{[1-3]}$. The best nozzle spacing and pipe spacing will also be determined in irrigation system planning, thereby, the equipment cost and energy consumption will be reduced as much as possible.

For the process of sprinkler irrigation with low pressure, the jet flow gets broken under various forces, such as aerodynamic force, inertia force, surface tension, etc. The existence of the breakup length has an important effect on the atomizing process, the length

Received date: 2016-08-12 Accepted date: 2017-08-20

Biographics: Yue Jiang, PhD, Assistant Research Fellow, research interests: irrigation theory and technical innovation, Email: jy261715267@126.com; Chao Chen, PhD, Assistant Research Fellow, research interests: design of water-saving irrigation equipment. Email: chch3605@ujs.edu.cn; Qingjiang Xiang, PhD, Associate Research Fellow, research interest: jet flow theory, Email: xiang_qj@163.com.

*Corresponding author: Hong $\mathbf{L i}, \mathrm{PhD}$, Research Fellow, research interests: design of water-saving irrigation equipment. Research Center of Fluid Machinery Engineering and Technology, Jiangsu University, 301 Xuefu Road, Zhenjiang 212013, Jiangsu, China. Tel: +86-13952891655, Email: hli@ujs.edu.cn. will affect the range and the irrigation uniformity. As the breakup of jet flow is influenced by many factors, the breakup mechanism remains a mystery. In recent years, more and more attention has been focused on it. Some researchers found that axisymmetrical and nonaxisymmetrical surface waves formed along the jet surface because of the interaction between gas and liquid phases. Some surface waves are unstable, they develop over time or space and ultimately lead to the breakup of jet flows. The distance from nozzle outlet to the first breakup point of jet flow is defined as jet breakup length ${ }^{[4-7]}$.

Both breakup process and jet breakup length can be obtained by theoretical analyses, experimental methods and numerical simulations. In many previous literatures ${ }^{[8-13]}$, the dispersion equation of jet was constructed by theory, and the results of numerical computation were presented. The instability of axisymmetrical and nonaxisymmetrical surface waves were analyzed, however, all these theoretical results are lack of sufficient experimental verification. In literature ${ }^{[14-20]}$, the breakup process of jet was observed using high speed photography, and the functional effect of jet velocity on jet breakup length was analyzed. In literatures ${ }^{[21-26]}$, the breakup process of jet was simulated using an algorithm based on the finite volume method and the volume of fluid (VOF) method, and the physics about the jet breakup and drop formation was captured. However, previous researches of jet focus on high pressure jet rather than low pressure jet which is used for irrigation sprinklers. The jet used in agricultural irrigation has a larger nozzle diameter and a longer range, which is different from the previous literatures, and the results may be totally different. Meanwhile, previous studies of jet breakup consider only one 
method separately, without mutual authentications among different methods.

Jet breakup length is the original and macro reflection of jet flow, and the sprinkler range is the final and macro reflection of jet flow. Establishing the relationship between two parameters can learn more about the spraying mechanism, and providing the theory basis for the research and development of new sprinklers. In this paper, the optimal jet breakup length will be determined by comparing the theoretical value with the experimental value and simulated value. A semi-empirical and semi-theoretical formula of sprinkler range would be deduced by considering the theoretical jet breakup lengths. Then the new formula would be compared with the previous empirical formulas in light of accuracy and reliability, they would provide the technical support to the sprinkler irrigation planning.

\section{Materials and methods}

\subsection{Theoretical analysis}

\subsubsection{Dispersion equation}

The cylindrical coordinate system $(r, \theta, z)$ was established according to the actual physical problems of jet, as presented in Figure 1. Assuming the jet flow direction is the same as the z-axis, and gas fluid and liquid fluid are incompressible flow without effects of temperature. $U_{0}$ is the initial velocity of jet symmetrically sprayed into still air, $\mathrm{m} / \mathrm{s} ; a$ is the radius of jet, $\mathrm{m} ; \rho_{g}$ and $\rho_{l}$ are the densities of gas and liquid, $\mathrm{kg} / \mathrm{m}^{3} ; \sigma$ is the coefficient of water surface tension; $\eta$ is the surface wave amplitude of gas-liquid interface, $\mathrm{m}$.

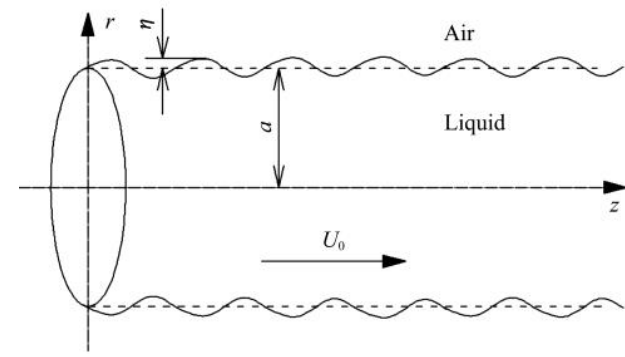

Figure 1 Diagram of the cylindrical jet and surface wave

Because of the influence of gas and liquid density ratio and the liquid surface tension, a pressure jump appears in the gas-liquid interface $(r=a)$, namely:

$$
\bar{P}_{l}=\bar{P}_{g}+\frac{\sigma}{a}
$$

As a result of the unavoidable disturbance in actual jet, the velocity and pressure of basic flow will be changed under disturbance velocity $u$ and pressure $p$, namely:

$$
\begin{gathered}
U_{j}=\bar{U}_{j}+u_{j} ; \quad u_{j}=\left(u_{j r}, 0, u_{j z}\right) \\
P_{j}=\bar{P}_{j}+p_{j}
\end{gathered}
$$

where, the overline represents the eigenvalue of basic flow, the subscript label $j=g$ or $l$ representing the gas phase or liquid phase, respectively.

Due to the instability of this study, normal mode method was used, such as:

$$
\left(u_{j}, p_{j}, \eta\right)=\left(\tilde{u}_{j}(r), \tilde{p}_{j}(r), \eta_{0}\right) e^{i k z+s t}, \quad(j=g, l)
$$

where, $\eta_{0}$ is initial amplitude of surface wave, $\mathrm{m} ; k$ is complex wave number; $s$ is complex growth rate.

Analyzing the perturbation pressure field, the instability dispersion equation of liquid jet can be calculated as:

$$
\frac{\rho_{l}\left(s+i k U_{0}\right)^{2}}{l} \frac{I_{0}(l a)}{I_{1}(l a)}-\frac{\rho_{g} s^{2}}{k} \frac{K_{0}(k a)}{K_{1}(k a)}+\frac{\sigma}{a^{2}}\left(1-a^{2} k^{2}\right)=0
$$

To illustrate the problem in general, Equation (5) should be dimensionless. Import the following parameters as:

$$
\begin{aligned}
& \alpha=k a, L=l a, S=\frac{s a}{U_{0}}, W_{e}=\frac{\rho_{l} U_{0}^{2} a}{\sigma}, R_{e}=\frac{\rho_{l} U_{0} a}{\mu}, Q=\frac{\rho_{g}}{\rho_{l}} \\
& L=\alpha \sqrt{\frac{R_{e}(S+i \alpha)}{R_{e}(S+i \alpha)+2 \alpha^{2}}}
\end{aligned}
$$

Then the final dimensionless equation was obtained as:

$$
\frac{(S+i \alpha)^{2}}{L} \frac{I_{0}(L)}{I_{1}(L)}-\frac{Q S^{2}}{\alpha} \frac{K_{0}(\alpha)}{K_{1}(\alpha)}+\frac{1-\alpha^{2}}{W_{e}}=0
$$

where, $\alpha$ represents dimensionless wave number; $S$ represents dimensionless growth gate; $W_{e}$ is Weber number; $R_{e}$ is Reynolds number; $Q$ is the ratio of gas and liquid densities; $I_{0}$ and $K_{0}$ are 0 -Bessel function of the first kind and 0-Bessel function of the second kind respectively; $I_{1}$ and $K_{1}$ are 1-Bessel function of the first kind and 1-Bessel function of the second kind respectively.

\subsubsection{Numerical calculation}

The dispersion equation of liquid jet represents the relationship between dimensionless wave number and dimensionless growth gate. Because the equation is a nonlinear complex equation, the analytic solution cannot be obtained directly. Matlab numerical software was used to solve the dispersion equation. If wave number $k$ is a real number and growth rate $s$ is a complex number, the disturbance is called as a temporal mode due to the characteristic of the rise in time and the oscillation in space; Otherwise, if $k$ is a complex number and $s$ is a purely imaginary number, the disturbance is called as a spatial mode due to the characteristic of the rise in space and the oscillation in time. According to Shi et al. ${ }^{[27]}$, the two modes give almost the same results under certain conditions and for convenience, temporal mode was adopted to analyze the jet process of fluid in this study.

Assuming that the jet flow gets broken when the liquid surface wave amplitude achieves the radius $a$ of the undisturbed jet, the jet breakup length is described as follows ${ }^{[28]}$ :

$$
L_{b}=\frac{U_{0}}{s_{\max }} \ln \frac{a}{\eta_{0}}
$$

where, $s_{\max }$ is the maximum perturbation growth rate; $\eta_{0}$ is concerned with the geometric factors of nozzle and it is determined by experiment.

\subsection{Experimental measurements}

\subsubsection{Measurement of sprinkler range}

To get a large number of accurate experimental data, this experiment was carried out in the Sprinkler Irrigation Experimental Hall, Jiangsu University in China. The open-type experimental site contains the standard rain gauges arranged radially all round and the advanced test instruments. In this research, the $\mathrm{PY}_{1} 15$ type sprinkler was adopted as prototype, in which the "15" refers to the inlet diameter of sprinklers, as presented in Figure 2a, and the $\mathrm{PY}_{1} 10$ type sprinkler and $\mathrm{PY}_{1} 20$ type sprinkler were used for validation. The elevation angles of nozzle are $30^{\circ}$, the mainly nozzle diameters of three sprinklers are $3 \mathrm{~mm}, 4 \mathrm{~mm}, 5 \mathrm{~mm}, 6 \mathrm{~mm}, 7 \mathrm{~mm}, 8 \mathrm{~mm}$ and $9 \mathrm{~mm}$, the working pressures are $150 \mathrm{kPa}, 200 \mathrm{kPa}, 250 \mathrm{kPa}, 300 \mathrm{kPa}$, $350 \mathrm{kPa}, 400 \mathrm{kPa}$ and $450 \mathrm{kPa}$.

Figure $2 \mathrm{~b}$ presents the structure sizes of nozzle, in which, $D_{1}$ is the outlet diameter of jet; $D_{2}$ is the shrinking entrance diameter of 
jet; $\theta$ is the cone angle of nozzle; $L$ is the total length of nozzle. Nine nozzle models with different structures were established and compared on their calculations, as presented in Table 1 .

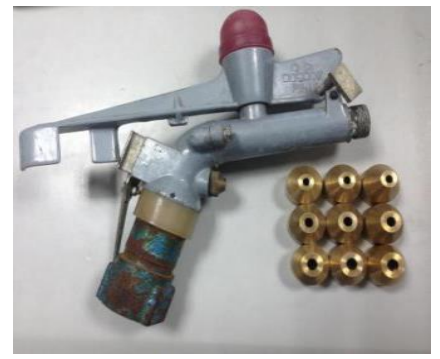

a. Prototype

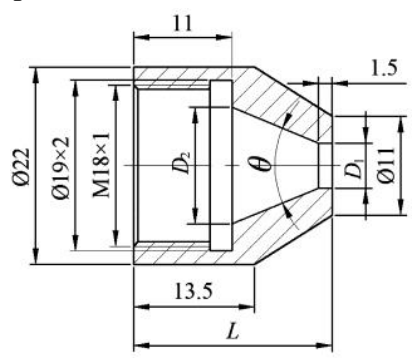

b. Nozzle structure
Figure 2 Prototype sprinkler of $\mathrm{PY}_{1} 15$ and nozzle structure

Table 1 Parameters of nine nozzles

\begin{tabular}{ccccc}
\hline Nozzle type & $D_{1} / \mathrm{mm}$ & $\theta /\left(^{\circ}\right)$ & $D_{2} / \mathrm{mm}$ & $L / \mathrm{mm}$ \\
\hline A & 4 & 35 & 13 & 26.81 \\
B & 4 & 45 & 13 & 23.35 \\
C & 4 & 55 & 13 & 21.16 \\
D & 5 & 35 & 13 & 25.22 \\
E & 5 & 45 & 13 & 22.14 \\
F & 5 & 55 & 13 & 20.21 \\
G & 6 & 35 & 13 & 23.64 \\
H & 6 & 45 & 13 & 20.94 \\
I & 6 & 55 & 13 & 19.24 \\
\hline
\end{tabular}

According to specification ${ }^{[29]}$, the experiment of nozzle range used the radial ray method. The rain gauges were arranged for 12 rays with a uniform angle using sprinkler as the center, and the equal space was $1.0 \mathrm{~m}$, as shown in Figure 3. The distance from the point with irrigation intensity of $0.3 \mathrm{~mm} / \mathrm{h}$ (the point with irrigation intensity of $0.15 \mathrm{~mm} / \mathrm{h}$ when the spray flow below $0.25 \mathrm{~m}^{3} / \mathrm{h}$ ) to the center of sprinkler was defined as the sprinkler range. The test time was $1 \mathrm{~h}$, and the experiment was repeated three times, then the values were averaged.

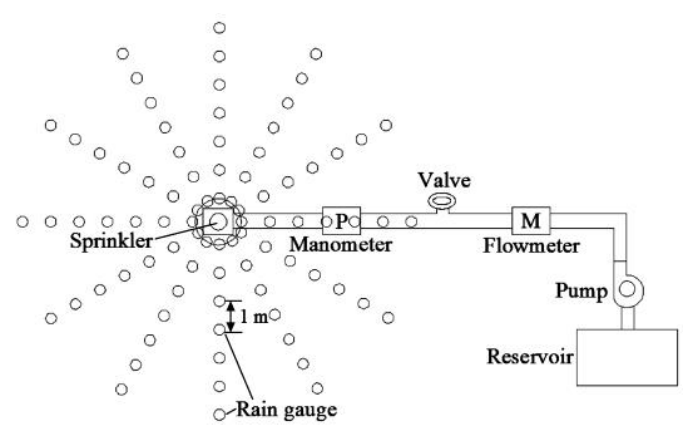

Figure 3 Indoor sprinkler test equipment layout

\subsubsection{Measurement of jet breakup length and initial amplitude}

The experimental was carried out for the sprinklers without rotation, and the experimental methods were divided into two kinds: shadow method and straight beam method. The shadow method was used to obtain the initial amplitude of surface wave $\left(\eta_{0}\right)$, and the straight beam method to measure the jet breakup length. Both experiment methods were performed using a high-speed camera with capable maximum frame rate of 150000 frames per second (fps), and it was set to $10000 \mathrm{fps}$ that is $0.1 \mathrm{~ms}$ for each frame in this test. The exposure time was set as $5 \mu \mathrm{s}$. For the experiment with shadow method, the optical system consisted of a $1000 \mathrm{~W}$ camera light and a piece of ground glass. The light went through the jet flow while flow fluctuates, then a shadow image of jet and surface wave was captured by the camera. The ground glass was used to attenuate the light. To capture more clear images, a $100 \mathrm{~mm}$ micro-lens was configured on the camera. The schematic experimental system is shown in Figure 4a. For the experiment with straight beam method, the optical system only includes a $1000 \mathrm{~W}$ camera light. A black curtain was placed at the back of the jet area, and the jet flow was illuminated by the light, placing horizontally at the capture zone. A tape was fixed on the black curtain and its track is the same as the trail of jet flow. To capture more clear images, a $50 \mathrm{~mm}$ prime lens was configured on the camera. The schematic experimental system is shown in Figure 4b.

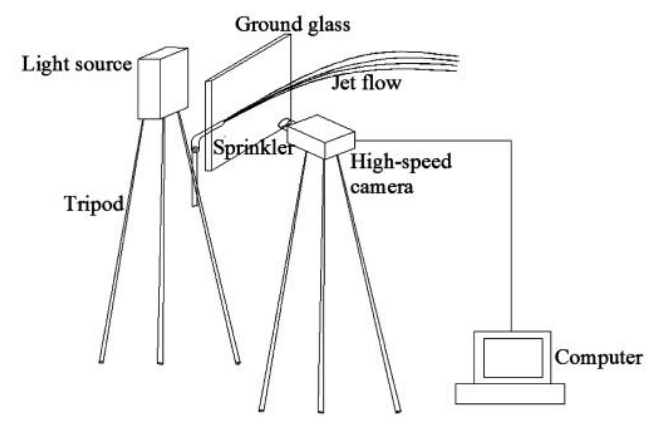

a. Schematic of jet surface wave measurement

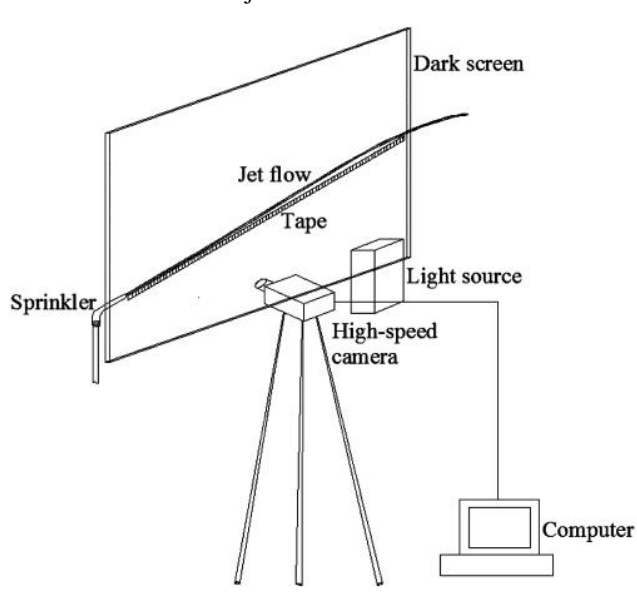

b. Schematic of jet breakup length measurement

Figure 4 Schematic of measurement for jet surface wave and jet breakup length

\subsubsection{Image processing}

Figure 5 presents the photo taken at the pressure of $400 \mathrm{kPa}$ with nozzle type $\mathrm{E}$ when the jet is stable. Both sides of the jet boundary were identified using the self-made Matlab image processing program and the least square method was used to fit the jet boundary as two straight lines. By identifying the surface wave peaks, the initial amplitude was obtained by calculating the distance between wave peak and boundary line. Figure 5 only shows the location of wave peaks on the upper side of jet flow.

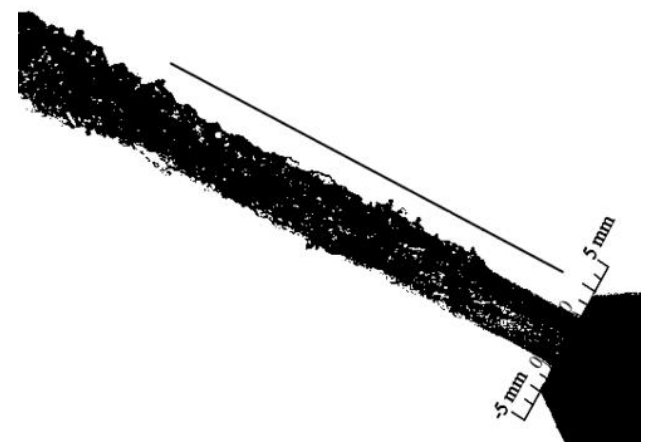

Figure 5 Visualization of jet surface wave 
Using the image measurement software, the initial amplitude was measured as $0.15 \mathrm{~mm}$ in Figure 5. For convenient calculation, the left and right boundaries of spray orifice were defined as zero point of the coordinate. The vertical upward direction of upper edge was defined as the positive direction while the vertical downward direction of lower edge was the negative.

\subsection{Numerical simulation}

For numerical simulation method, the ANSYS ICEM software was used to observe the jet breakup length. The geometry and meshing is shown in Figure 6. Meanwhile, the grid independence was evaluated by adopting four grid sizes for the computational domain at the pressure of $400 \mathrm{kPa}$ with nozzle type E. The results are shown in Table 2. The grid independence on the difference in breakup length between No. 2 and No.4 grid was less than $0.7 \%$.

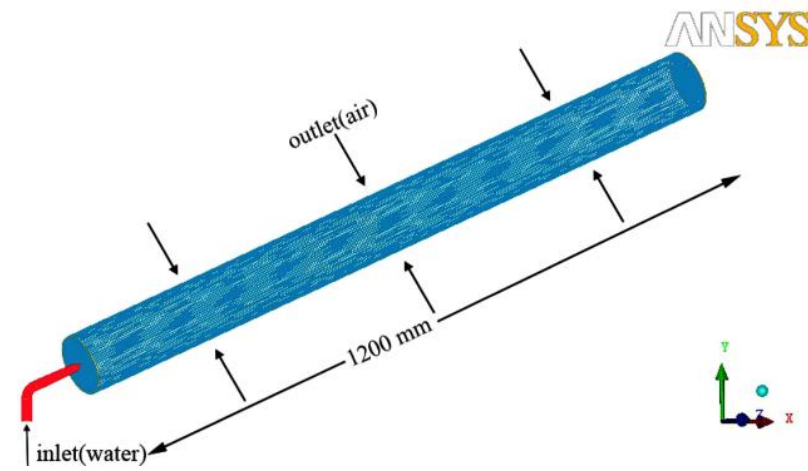

Figure 6 3D view of the simulation sections

Table 2 Grid independence analysis results for type $\mathbf{E}$ nozzle when $p=400 \mathrm{kPa}$

\begin{tabular}{ccccc}
\hline Grid No. & 1 & 2 & 3 & 4 \\
\hline Number of grid $/ 10^{6}$ & 40.7 & 63.5 & 100.1 & 150.8 \\
Breakup length $/ \mathrm{mm}$ & 671.5 & 642.4 & 640.7 & 637.5 \\
\hline
\end{tabular}

\subsubsection{Governing equations}

At present, there are two main ways to solve the interface problem of the interaction between two fluids: the Level Set method and the VOF method, which are both Euler methods. The Level Set method is suitable for solving the interface curvature problem, while the VOF method is simpler and is able to maintain the conservation of fluid volume in calculation. VOF method can also avoid the mass non-conservation which exists in Level Set method, however, the solution of normal direction and precision of curvature can be optimized by using Level Set method. Therefore, the basic idea of CLSVOF method which combines two methods was proposed by some scholars recently. This study will discuss the spatial breakup characteristics of the core section of low-pressure jet by using the CLSVOF method.

The continuity equation, momentum equation and energy equation have been used to serve as control equations to solve problems in gas-liquid two-phase flow process. Without considering the influence of temperature on the flow, the control equations of single phase and two-phase flow are written in a uniform form, as follows:

$$
\begin{gathered}
\nabla \cdot \boldsymbol{u}=0 \\
\rho(\phi)\left(\frac{\partial \boldsymbol{u}}{\partial t}+\boldsymbol{u} \cdot \nabla \boldsymbol{u}\right)=-\nabla p+\rho(\phi) \boldsymbol{g}-\sigma \kappa(\phi) \nabla H(\phi)+\nabla \cdot \boldsymbol{\tau} \\
\boldsymbol{\tau}=2 \mu(\phi) \boldsymbol{S}
\end{gathered}
$$

where, $\boldsymbol{u}$ is velocity; $p$ is pressure; $g$ is gravitational acceleration; $\sigma$ is surface tension coefficient; $\tau$ is viscous stress tensor; $\mu(\phi)$ is viscosity; $\boldsymbol{S}$ is strain rate tensor; $\rho(\phi)$ is density; $\phi$ is Level Set function; $\sigma \kappa(\phi) \nabla H(\phi)$ is surface tension term, which can couple the surface tension to momentum equation as a body force; $\kappa(\phi)$ is curvature and $H(\phi)$ is Heaviside function, which is to make the control equations of single phase flow and mixed phase flow in a uniform form.

The form of Heaviside function could be expressed as

$$
H(\phi)=\left\{\begin{array}{cl}
1 & (\phi>+h) \\
0 & (\phi<-h) \\
0.5+\phi /(3 h)+\sin [2 \pi \phi /(3 h)] /(2 \pi) & (|\phi| \leq h)
\end{array}\right.
$$

where, $h$ is mesh size. The mathematics of strain rate tensor $(\boldsymbol{S})$ could be expressed as

$$
\boldsymbol{S}=\frac{1}{2}\left[(\nabla \boldsymbol{u})+\left(\nabla \boldsymbol{u}^{T}\right)\right.
$$

The value of different areas of $\rho(\phi)$ and $\mu(\phi)$ were calculated by the Heaviside function and they could be expressed as

$$
\left\{\begin{array}{l}
\rho(\phi)=\rho_{l}(1-H(\phi))+\rho_{g} H(\phi) \\
\mu(\phi)=\mu_{l}(1-H(\phi))+\mu_{g} H(\phi)
\end{array}\right.
$$

\subsubsection{Numerical procedure}

The governing equations were solved by computational fluid dynamic software ANSYS FLUENT 14.0 based on associated boundary and initial conditions. The finite volume method was applied to discretize the governing equations in the computational domain. The RNG $k-\varepsilon$ turbulent model was applied to solve the unsteady calculation. Level Set-VOF method model was selected as a multiphase model. The SIMPLE algorithm considering both velocity and pressure was adopted, including a PRESTO! scheme for discretization of pressure equation, a Geo-Reconstruct scheme for discretization of volume fraction equation and a second order upwind scheme for discretization of other equations. Structured grids were used for meshing the solution domain. The time step $(t)$ was set to $10^{-5} \mathrm{~s}$. In order to obtain high accuracy data, the residual sum was computed and set for each iteration and the convergence criterion was less than $10^{-5}$ for all equations.

\subsection{Known empirical formulas for range}

Four empirical formulas of range applied home and abroad would be chosen to compare with the formulas in this paper, i.e.:

Cauazza formula ${ }^{[30]}$ :

Chang W H formula ${ }^{[31]}$.

$$
R=1.35 \sqrt{D p}
$$

$$
R=1.70 D^{0.487} p^{0.45}
$$

Jia W L formula ${ }^{[32]}$

$$
R=0.415 D \sqrt[3]{\frac{\alpha}{\pi} \times 1.8 \times 10^{5}}\left(\frac{p}{10^{4}}\right)^{2 / 3}
$$

Feng C D formula ${ }^{[33]}$ :

$$
R=4 \mu^{2} p \sin ^{2} \alpha\left(\cot \alpha-0.216 \frac{p^{0.94}}{D}\right)
$$

Gan Z M formula ${ }^{[34]}$ :

$$
R=\xi p^{m} D^{n}
$$

where, $R$ is the sprinkler range, $\mathrm{m} ; D$ is the nozzle diameter, $\mathrm{m} ; p$ is the working pressure, $\mathrm{kPa} ; \alpha$ is the elevation angle of nozzle, $\left({ }^{\circ}\right)$; $\mu$ is the kinematic viscosity, $\mathrm{m}^{2} / \mathrm{s}$; $\xi$ is the coefficient.

\section{Results and discussion}

\subsection{Experimental results of ranges}

Table 3 shows the experimental results of sprinkler range. As expected, the sprinkler ranges increase with the increase of pressure for the same nozzle diameter and nozzle cone angle. For the same 
nozzle diameter and pressure, the ranges decrease with the increase of nozzle cone angle. For the same nozzle cone angle and pressure, the ranges increase with the increase of the nozzle diameter.

Table 3 Experimental results of sprinkler range

\begin{tabular}{cccccccc}
\hline \multirow{2}{*}{$\begin{array}{c}\text { Sprinkler } \\
\text { type }\end{array}$} & $\begin{array}{c}\text { Nozzle } \\
\text { diameter } \\
/ \mathrm{mm}\end{array}$ & $\begin{array}{c}\text { Nozzle } \\
\text { cone } \\
\text { angle } /\left(^{\circ}\right)\end{array}$ & 200 & 250 & 300 & 350 & 400 \\
\cline { 6 - 8 } & 4 & 35 & 14.1 & 14.5 & 15.8 & 16.2 & 16.5 \\
& 4 & 45 & 13.4 & 14.1 & 15 & 15.4 & 15.8 \\
& 4 & 55 & 13.2 & 14 & 14.9 & 15.1 & 15.6 \\
& 5 & 35 & 15.2 & 15.7 & 16.5 & 17 & 17.5 \\
$\mathrm{PY}_{1} 15$ & 5 & 45 & 14.9 & 15.5 & 16.1 & 16.7 & 17.2 \\
& 5 & 55 & 14.5 & 15.3 & 15.9 & 16 & 16.7 \\
& 6 & 35 & 15.1 & 15.6 & 16.2 & 17.2 & 18.1 \\
& 6 & 45 & 14.8 & 15.3 & 16.1 & 17 & 17.7 \\
& 6 & 55 & 14.5 & 15.2 & 16 & 17 & 17.5 \\
\hline
\end{tabular}

3.2 Theoretical results of jet breakup lengths

3.2.1 Effect of initial velocity, nozzle radius and cone angle on jet breakup instability

In the dispersion equation, the $R_{e}$ and $W_{e}$ are proportional to velocity, so the change of initial velocity will cause the change of $R_{e}$ and $W_{e}$, thus cause the instability of jet flow. Figure $7 \mathrm{a}$ presents the influence of jet initial velocities on the breakup process with nozzle radius $2.5 \mathrm{~mm}$ and cone angle $45^{\circ}$. With the increase of initial velocity, the maximum perturbation growth rate and unstable wave numbers decrease, which means when the jet is in a breakup mode, a higher jet initial velocity would prevent the breakup of jet, and that is totally different from the atomization situation of high speed jet.

When the velocity is constant, the variation of nozzle radius will cause the change of $R_{e}$ and $W_{e}$, thus causing the instability of jet flow. Figure $7 \mathrm{~b}$ presents the calculation example of jet breakup with an initial velocity of $16.87 \mathrm{~m} / \mathrm{s}$ and a cone angle of $45^{\circ}$. The maximum perturbation growth rate and unstable wave numbers decrease with increasing nozzle radius, which means as nozzle radius increases, the breakup of jet may be suppressed, namely, the jet is in a breakup mode.

Figure $7 \mathrm{c}$ presents the influence of nozzle cone angle on the breakup process with nozzle radius $2.5 \mathrm{~mm}$ and working pressure $200 \mathrm{kPa}$. For the same pressure, the jet velocity changed with different cone angles. The maximum perturbation growth rate and unstable wave numbers increase with the increasing of nozzle cone angle. That is because the total length of nozzle decreases with the increasing of nozzle cone angle, and the flow instability is intensified.

\subsubsection{Determination of the jet breakup lengths}

According to the calculation of Equation (7), Figure 8 presents the relationship between the jet breakup length and jet velocity for different nozzle types. Seen from the figure, with the increase of jet velocity or nozzle diameter, the jet breakup length increases. This is because that the maximum perturbation growth rate is small when the jet velocity is low, and the perturbation develops slowly along the jet flow direction. There is a long distance before the perturbation approaches the axis of jet, which indicates that the effect of perturbation on jet breakup length is weaker than the effect of jet velocity at that time, namely, the jet breakup length increases with higher jet velocity. For the same jet velocity and nozzle diameter, the jet breakup length decreases with the increasing of cone angle, it shows good consistency with the phenomenon which the flow instability would be intensified with the increasing of nozzle cone angle.

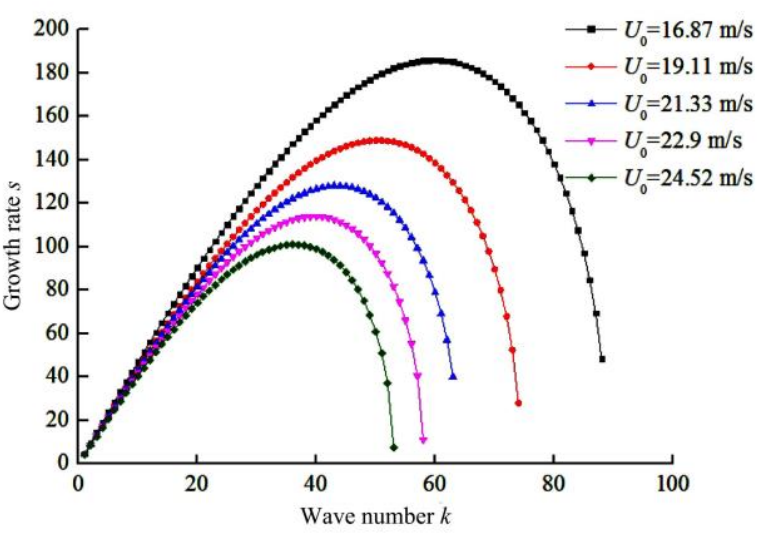

a. Effect of jet velocity on liquid jet

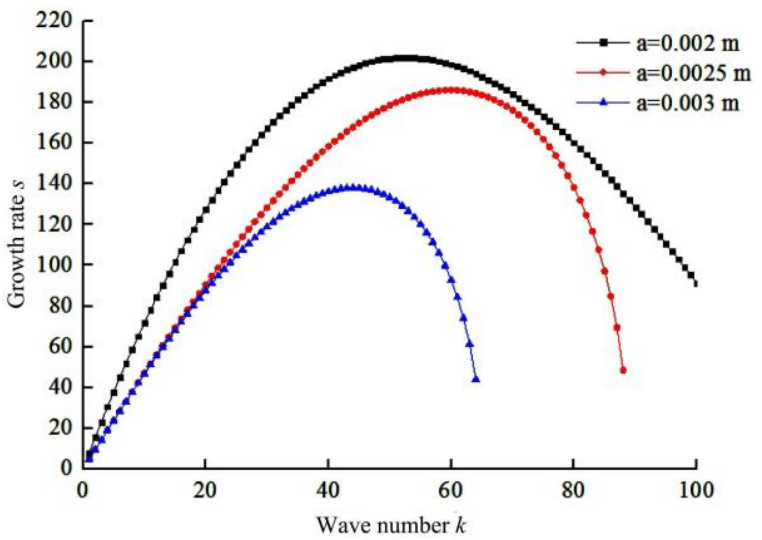

b. Effect of nozzle radius on liquid jet

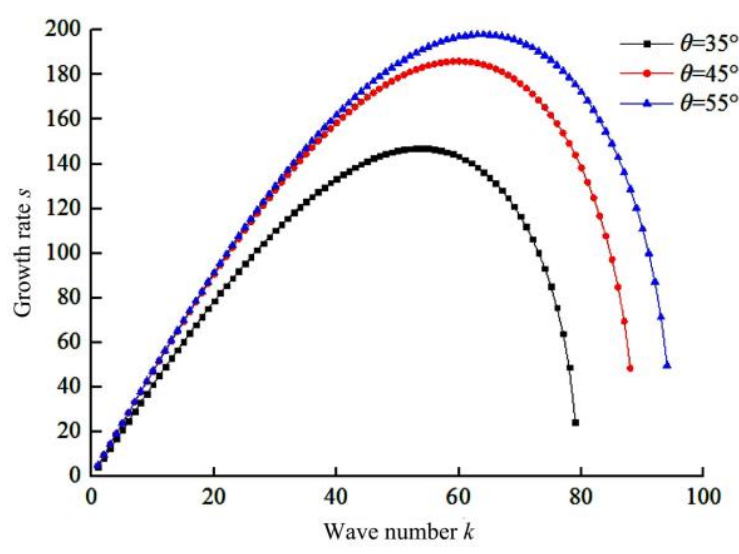

c. Effect of nozzle cone angle on liquid jet

Figure 7 Effects of jet velocity, nozzle radius and nozzle cone angle on liquid jet

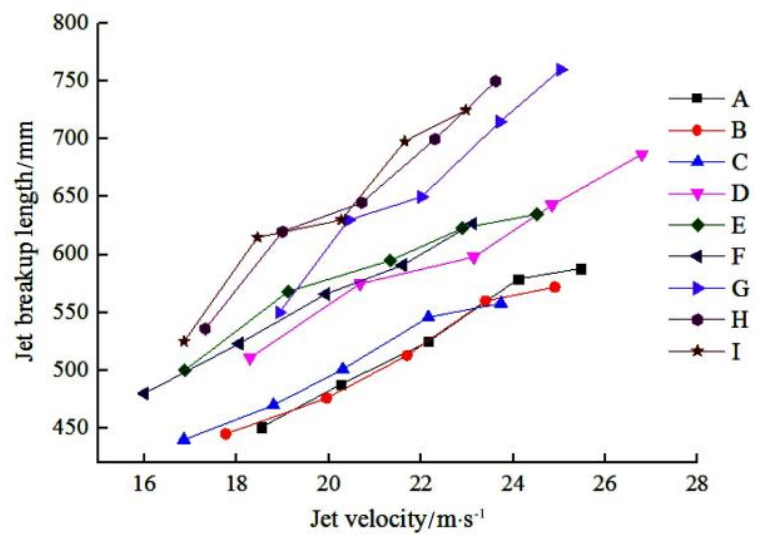

Figure 8 Relationship between jet breakup length and jet velocity 


\subsection{Experimental value of jet breakup lengths}

With the entrainment of air into the jets, the amount of fluid increases as the jet moves continuously and flow friction increases as the jet boundary extends to the sides. Due to the principle of jet and static flow mixture, the jet starts to fracture and break up. Different photographs were captured for this analysis. Figure 9 presents the breakup process of jet at pressure $200 \mathrm{kPa}, 300 \mathrm{kPa}$ and $400 \mathrm{kPa}$ with type $\mathrm{E}$ nozzle. In these figures, the jet direction is from left to right and the start measuring position of tape is $10 \mathrm{~cm}$. The jet breakup section is marked by white circle. With elevated pressure, the jet breakup length increased, which indicated that the range of jet would be longer with a higher pressure. The lengths for the three pressures are about $450 \mathrm{~mm}, 580 \mathrm{~mm}$ and 600 $\mathrm{mm}$, respectively.

\subsection{Numerical simulation of jet breakup lengths}

Contours of liquid volume fraction of the jet are illustrated in Figure 10 by using the sprinkler with type E nozzle under three different pressures. The lengths of the jet breakup section were analyzed using ScanIt software. The result shows that jet breakup length increases with the working pressure. The simulation lengths are $510.1 \mathrm{~mm}, 606.8 \mathrm{~mm}$ and $642.4 \mathrm{~mm}$, respectively.
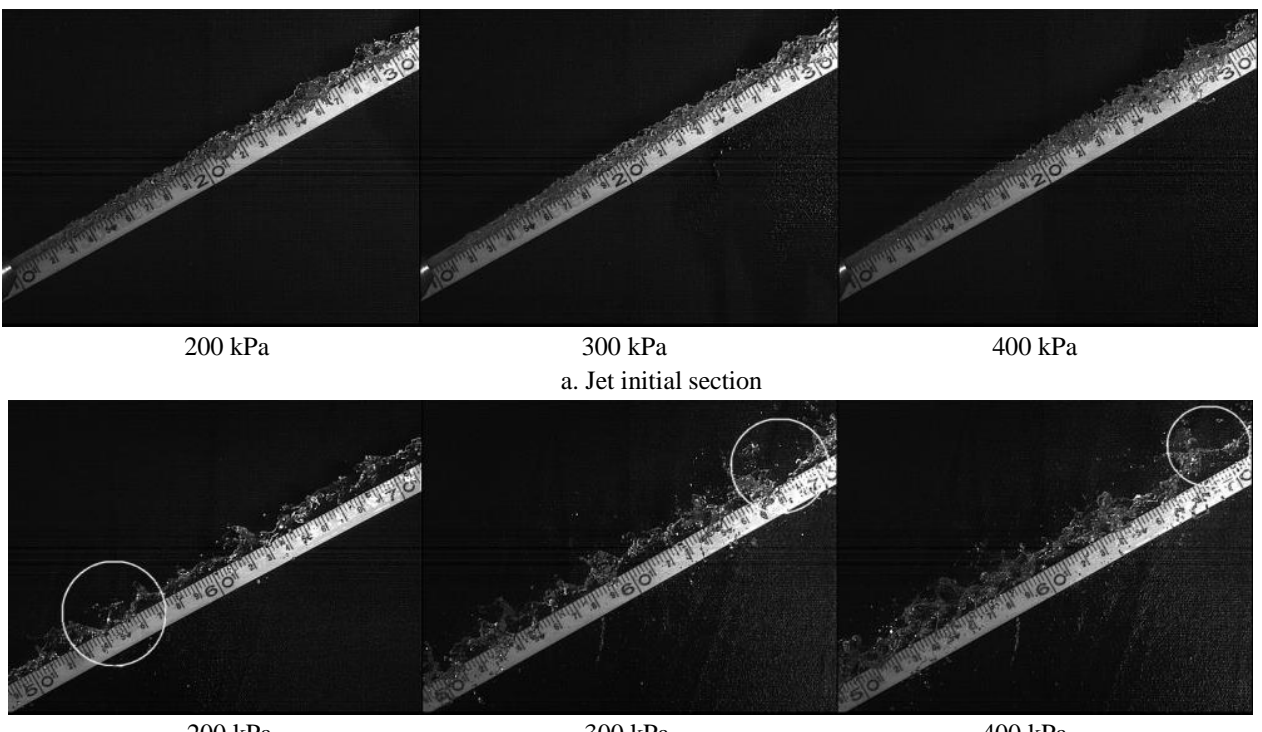

$200 \mathrm{kPa}$

$300 \mathrm{kPa}$

$400 \mathrm{kPa}$

b. Jet breakup section

Figure 9 Photographs of jet under different pressures

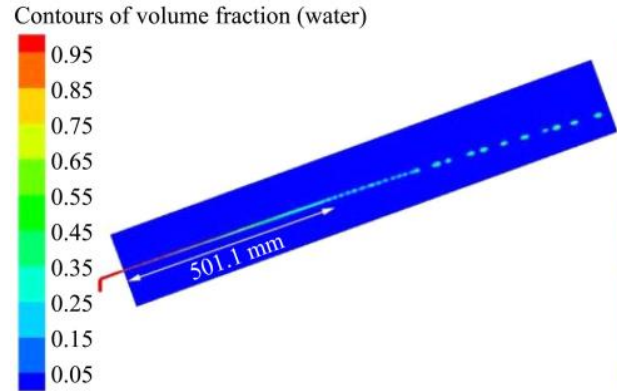

a. $200 \mathrm{kPa}$

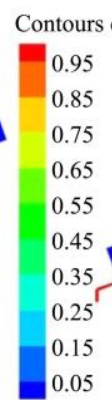

0.05

b. $300 \mathrm{kPa}$

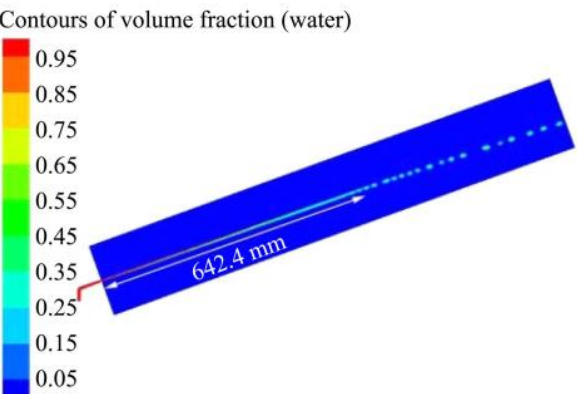

c. $400 \mathrm{kPa}$

Figure 10 Contours of liquid volume fraction with type E nozzle under different pressures

\subsection{Comparison between theory, experiment and numerical simulation}

Table 4 presents the comparison among the three approaches under different pressures and nozzle types. It shows that, for the sprinkler with different nozzle types and pressures, the experimental jet breakup lengths are the lowest and the simulation values are the highest. The reason for this is that the simulation process neglects the influence of external factors on jets which exists actually in the experiment; the external factors such as winds, pressure fluctuation of water, and processing stage of nozzle would decrease the breakup length in the experiment. However, there is a good agreement of the three results with an error of less than $10 \%$ for jet breakup lengths, which validates the accuracy and reliability of theoretical results, experimental data and simulation results. Furthermore, the theoretical value is closer to the average value, which suggests that the theoretical calculation method could accurately calculate the jet breakup length.

\subsection{Calculation formula of range}

Considering the influence of jet breakup length on the range of jet, the formula of range was fit out by using the least square method, and the relative regression coefficients was obtained.

$$
R=0.7273\left(\frac{U_{0}}{s_{\max }} \ln \frac{a}{\eta_{0}}\right)^{0.4831}
$$

\subsection{Precision and applicability of formulas}

\subsubsection{Comparison of calculated values and measured values}

The formula in this paper is the curve fitting formula, there are some errors between the calculation and the measured values inevitably. The relative errors between the measured values and the calculation for range under the pressure of $200 \mathrm{kPa}, 300 \mathrm{kPa}$ and $400 \mathrm{kPa}$ are reported in Table 5. From the table, the relative errors between the fitted values and the actual values, with one or two exceptions are less than $2.5 \%$, which means the fitting precision of the formula to measured values is satisfactory. 
Table 4 Comparison of jet breakup lengths with different pressures and nozzle types

\begin{tabular}{|c|c|c|c|c|c|c|c|c|c|c|c|c|}
\hline \multirow{3}{*}{$\begin{array}{l}\text { Nozzle } \\
\text { type }\end{array}$} & \multicolumn{12}{|c|}{ Working pressure $/ \mathrm{kPa}$} \\
\hline & \multicolumn{4}{|c|}{200} & \multicolumn{4}{|c|}{300} & \multicolumn{4}{|c|}{400} \\
\hline & T.V & E.V & S.V & AVG & T.V & E.V & S.V & AVG & T.V & E.V & S.V & AVG \\
\hline A & 451 & 435 & 476 & 454 & 525 & 510 & 551 & 529 & 588 & 560 & 619 & 589 \\
\hline B & 445 & 420 & 470 & 445 & 513 & 505 & 543 & 520 & 572 & 565 & 599 & 579 \\
\hline $\mathrm{D}$ & 511 & 500 & 527 & 513 & 598 & 582 & 613 & 598 & 687 & 667 & 699 & 684 \\
\hline $\mathrm{E}$ & 500 & 450 & 510 & 487 & 595 & 580 & 607 & 594 & 635 & 600 & 642 & 626 \\
\hline $\mathrm{F}$ & 480 & 465 & 505 & 483 & 566 & 550 & 577 & 564 & 627 & 615 & 642 & 628 \\
\hline $\mathrm{G}$ & 550 & 520 & 560 & 543 & 650 & 635 & 678 & 654 & 760 & 740 & 780 & 760 \\
\hline $\mathrm{H}$ & 536 & 515 & 550 & 534 & 645 & 625 & 662 & 644 & 750 & 730 & 765 & 748 \\
\hline
\end{tabular}

Note: "T.V" represents "Theoretical value", "E.V" represents "Experimental value", "S.V" represents "Simulation value" and "AVG" represents average value.

Table 5 Comparison of measured data and predicted values for range

\begin{tabular}{|c|c|c|c|c|c|c|c|c|c|}
\hline Measured values/m & 14.1 & 13.4 & 13.2 & 15.1 & 14.9 & 14.8 & 15.1 & 14.8 & 14.5 \\
\hline Predicted values/m & 13.92 & 13.84 & 13.76 & 14.79 & 14.64 & 14.35 & 15.33 & 15.14 & 14.79 \\
\hline Relative error/\% & 1.20 & 3.28 & 4.27 & 2.65 & 1.73 & 0.99 & 1.53 & 2.31 & 2.38 \\
\hline Measured values/m & 15.8 & 15 & 14.9 & 16.6 & 16.1 & 16 & 16.2 & 16.1 & 16 \\
\hline Predicted values $/ \mathrm{m}$ & 15.18 & 14.82 & 14.66 & 15.96 & 15.93 & 15.54 & 16.17 & 16.44 & 16.37 \\
\hline Relative error $/ \%$ & 3.94 & 1.17 & 1.62 & 3.24 & 1.08 & 2.23 & 0.21 & 2.01 & 2.31 \\
\hline Measured values/m & 16.5 & 15.8 & 15.6 & 17.6 & 17.2 & 17 & 18.1 & 17.7 & 17.5 \\
\hline Predicted values/m & 16.39 & 15.62 & 15.44 & 17.07 & 17.29 & 16.33 & 17.92 & 17.81 & 17.52 \\
\hline Relative error/\% & 0.69 & 1.11 & 1.03 & 2.45 & 0.52 & 2.19 & 0.97 & 0.61 & 0.12 \\
\hline
\end{tabular}

3.7.2 Comparison between this formula and other empirical formulas

Figure 11 shows the relationship between nozzle diameter and sprinkler range for the nozzle with cone angle $45^{\circ}$ under the pressure of $300 \mathrm{kPa}$. Figure 12 shows the relationship between pressure and sprinkler range for the nozzle with nozzle radius $2.5 \mathrm{~mm}$ and cone angle $45^{\circ}$. From the results it is known that the new formula is in good agreement with the data of different types of sprinklers comparing with other empirical formulas, and the relative error is only $2.5 \%$. Comparing the measured values with the calculated data from the Cauazza formula, Chang W. H formula, Jia W. L formula, Feng C. D formula and Gan Z. M formula, and the relative errors are $6.5 \%, 7.4 \%, 30.1 \%, 13.9 \%$ and $10.6 \%$.

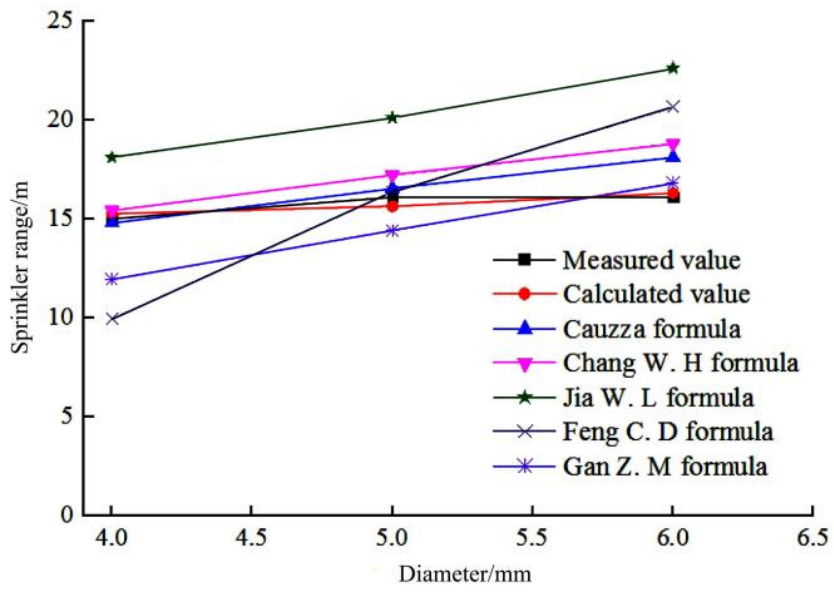

Figure 11 Relationship between nozzle diameter and sprinkler range

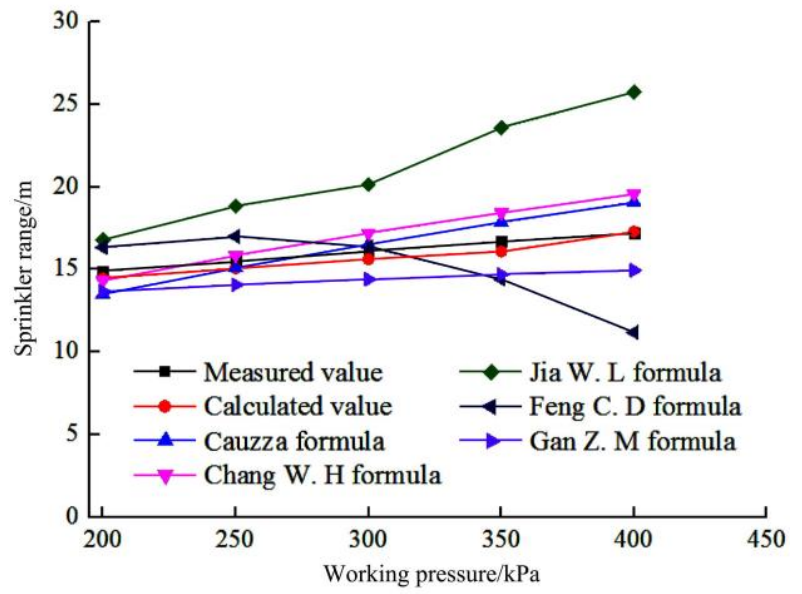

Figure 12 Relationship between pressure and sprinkler range

With higher pressures, the trend of calculated data from Feng C. D formula increases firstly then decreases and shows a parabola type, and it has a big relative error. With the increase of nozzle diameters and pressures, the calculated data of other formulas increases constantly and the power exponent curves are presented. The calculated values of Jia W. L formula are larger than the measured values, which the relative error goes beyond the range of allowable error. Instead, the calculated values of Gan Z. M formula are smaller than the measured values, which the error is about $10 \%$. The Cauazza formula and Chang W. $\mathrm{H}$ formula have the little relative errors, but the scope of application is limited due to the two formulas only be affected by nozzle diameter and pressure. Comparison shows that the fitting formula in this paper is in good agreement with the measured values and it is the best formula among all formulas in this article. 


\subsubsection{Verification of this formula for other sprinklers}

The formulas in this paper were obtained based on the measuring of the $\mathrm{PY}_{1} 15$ type sprinkler. To verify the suitability of formulas to other type sprinklers, $\mathrm{PY}_{1} 10$ type sprinkler and $\mathrm{PY}_{1} 20$ type sprinkler were used in comparison.

As the Table 6 shows, the relative errors between the fitted values and the measured values are less than $5 \%$. The formula of range presents in this article is in good agreement with the range of same series of sprinklers, which shows the new formula is more prevalent in application.

Table 6 Comparison of fitted values and other types sprinkler for range

\begin{tabular}{|c|c|c|c|c|c|c|}
\hline $\begin{array}{l}\text { Sprinkler } \\
\text { type }\end{array}$ & $\begin{array}{l}\text { Nozzle } \\
\text { diameter } \\
\text { /mm }\end{array}$ & $\begin{array}{c}\text { Nozzle } \\
\text { cone angle } \\
/\left(^{\circ}\right)\end{array}$ & $\begin{array}{c}\text { Pressure } \\
/ \mathrm{kPa}\end{array}$ & $\begin{array}{c}\text { Formula } \\
\text { calculation } \\
\text { /m }\end{array}$ & $\begin{array}{c}\text { Measured } \\
\text { value/m }\end{array}$ & $\begin{array}{l}\text { Relative } \\
\text { error/\% }\end{array}$ \\
\hline \multirow{12}{*}{$\mathrm{PY}_{1} 10$} & 3 & 35 & 150 & 10.12 & 10.5 & 3.62 \\
\hline & 3 & 35 & 300 & 12.01 & 11.7 & 2.65 \\
\hline & 3 & 45 & 150 & 10.16 & 10.1 & 0.59 \\
\hline & 3 & 45 & 300 & 11.83 & 11.3 & 4.69 \\
\hline & 3 & 55 & 150 & 9.51 & 9.7 & 1.95 \\
\hline & 3 & 55 & 300 & 10.55 & 10.7 & 1.40 \\
\hline & 4 & 35 & 200 & 11.34 & 11.5 & 1.39 \\
\hline & 4 & 35 & 350 & 12.20 & 12.7 & 3.93 \\
\hline & 4 & 45 & 200 & 11.52 & 11.3 & 1.95 \\
\hline & 4 & 45 & 350 & 12.51 & 12.2 & 2.54 \\
\hline & 4 & 55 & 200 & 10.83 & 11 & 1.54 \\
\hline & 4 & 55 & 350 & 12.22 & 12 & 1.83 \\
\hline \multirow{16}{*}{$\mathrm{PY}_{1} 20$} & 6 & 35 & 250 & 16.88 & 17.1 & 1.28 \\
\hline & 6 & 35 & 450 & 19.51 & 19.3 & 1.09 \\
\hline & 6 & 45 & 250 & 16.55 & 17 & 2.65 \\
\hline & 6 & 45 & 450 & 18.25 & 19 & 3.95 \\
\hline & 7 & 45 & 250 & 18.00 & 17.7 & 1.69 \\
\hline & 7 & 45 & 450 & 20.11 & 20.9 & 3.78 \\
\hline & 7 & 55 & 250 & 17.75 & 17.5 & 1.43 \\
\hline & 7 & 55 & 450 & 20.8 & 20.5 & 1.46 \\
\hline & 8 & 35 & 250 & 19.01 & 18.8 & 1.12 \\
\hline & 8 & 35 & 450 & 22.11 & 23 & 3.87 \\
\hline & 8 & 45 & 250 & 18.21 & 19 & 4.15 \\
\hline & 8 & 45 & 450 & 22.98 & 22.5 & 2.13 \\
\hline & 9 & 45 & 250 & 20.11 & 20.1 & 0.05 \\
\hline & 9 & 45 & 450 & 23.90 & 23.5 & 1.7 \\
\hline & 9 & 55 & 250 & 19.52 & 19.6 & 0.41 \\
\hline & 9 & 55 & 450 & 21.97 & 23.1 & 4.89 \\
\hline
\end{tabular}

\section{Conclusions}

In this study, the jet breakup lengths of low pressure liquid with different nozzle parameters were analyzed through theoretical calculation, experiments and numerical simulation. A semi-empirical and semi-theoretical formula of range for the rotating sprinkler was constructed by the curve fitting method and the formula was verified. The following can be drawn throughout the paper:

(1) The dispersion equation of cylindrical jet was established upon the linear instability theory. Within the discussed jet velocity and working pressure range, the numerical analysis showed that the maximum perturbation growth rate and unstable wave numbers decrease and jet breakup length increases with the increasing of nozzle radius and jet initial velocity. With the increase of nozzle cone angle, the maximum perturbation growth rate and unstable wave numbers increase and the jet breakup length decreases.

(2) The theoretical analysis gives a reasonable explanation to the influence of jet velocity, nozzle diameter and nozzle cone angle on jet breakup length. Comparing the experimental value with simulated value, the experimental jet breakup lengths are the lowest while the simulation values are the highest. Even though, the three results accord one another, and the relative error is less than $10 \%$, furthermore, the theoretical value is closer to the average value than the other two values.

(3) By introducing the theoretical calculation of jet breakup length, a semi-empirical and semi-theoretical formula of range for the rotating sprinkler was concluded by the curve fitting method. The high accuracy of the ranges determined by this formula and the average relative error was less than $2.5 \%$. The new formula is in good agreement with the data of different sprinkler types comparing with other empirical formulas, and the error was less than $5 \%$, which is greatly below that of other formulas. Further research should concentrate on the application of new formula for designing more types of sprinklers.

\section{Acknowledgments}

We acknowledge that this work was financially supported by the National Natural Science Foundation of China (No.51679109), the Natural Science Foundation of Jiangsu Province (BK20170555), the Natural Science Foundation of the Higher Education Institutions of Jiangsu Province (17KJB470001), Special Fund for Ago-scientific Research in the Public Interest of China (201503130), and the Project Funded by the Priority Academic Program Development of Jiangsu Higher Education Institutions (PAPD).

\section{[References]}

[1] Li J, Kawano H. Simulating water-drop movement from noncircular sprinkler nozzles. Journal of Irrigation Drainage Engineering, 1995; 121(2): 152-158.

[2] Han S, Evans R G, Kroeger M W. Sprinkler distribution patterns in windy conditions. Transactions of the ASAE, 1994; 37(5): 1481-1489.

[3] Seginer I, Kantz D, Nir D. The distortion by wind of the distribution patterns of single sprinklers. Agricultural Water Management, 1991; 19: 341-359.

[4] Turn M R, Healey J J, Sazhin S S. Stability analysis and breakup length calculations for steady planar liquid jets. Journal of Fluid Mechanics, 2011; 668: 384-411.

[5] Uchiyama Y, Abe Y, Kaneko A. Experimental study on influence of interfacial behavior on jet surface fragmentation. Proceedings of the 17th International Conference on Nuclear Engineering, 2009; 4: 433-442.

[6] Taveb R, Sakib M N, Ali M. Both experimental and numerical investigation on breakup length of cylindrical falling jet. Process Engineering, 2013; 56: 462-467.

[7] Jiang Y, Li H, Xiang Q J. Experiment on breakup process of low-pressure jets with different nozzle parameters and pressures. Transactions of the CSAM, 2015; 46(3):78-82.

[8] Reitz D B. Mechanism of atomization of a liquid jet. Physics of Fluids A, 1982; 25: 1730-1742.

[9] Hou J, Cao J M, Li G H. Derivation on linear stability theory of zero order dispersion relation in liquid jets. Journal of Chang'an University, 2011; 31(4): 94-97.

[10] Du Q, Guo J, Meng Y L. The instability analysis of gas rotations on the breakup of an annular liquid jet. Transactions of the CSICE, 2007; 25(3): 217-222.

[11] Park H, Yoon S S, Heister S D. On the nonlinear stability of a swirling liquid jet. International Journal of Multiphase Flow, 2006; 32(9): 1100-1109. 
[12] Ibrahim A A, Jog M A. Nonlinear breakup of a coaxial liquid jet in a swirling gas stream. Physics of Fluids, 2006; 18(11): 1141-1501.

[13] Wang X Y, Wang J F, Zhi L Z. Theory and experiment on jet breakup length of charged liquid. Transactions of the CSAM, 2013; 44(2): 93-96. (in Chinese)

[14] Wan Y X, Huang Y, Zhu Y. Experiment on the breakup process of free round liquid jet. Journal of Aerospace Power, 2008; 23(2): 208-214. (in Chinese)

[15] Zhu Y, Wan Y X, Huang Y. Study on the breakup lengths of free round liquid jets. Journal of Aerospace Power, 2007; 22(8): 1258-1263. (in Chinese)

[16] Morozumi Y, Fukai J. Growth and structure of surface disturbances of a round liquid jet in a coaxial airflow. Fluid Dynamics Research, 2004; 34 : 217-231

[17] Sallam K A, Dai Z, Faeth G M. Liquid breakup at the surface of turbulent round liquid jets in still gases. International Journal of Multiphase Flow, 2002; 28: 427-449.

[18] Wang F J, Fang T G. Liquid jet breakup for non-circular orifices under low pressures. International Journal of Multiphase Flow, 2015; 72: 248-262.

[19] Negeed E S R, Hidaka S, Kohno M. Experimental and analytical investigation of liquid sheet breakup characteristics. International Journal of Heat and Fluid Flow, 2011; 32(1): 95-106

[20] Davanlou A, Lee J D, Basu S. Effect of viscosity an surface tension on breakup and coalescence of bicomponent sprays. Chemical Engineering Science, 2015; 131: 243-255.

[21] Jin Z X, Dong Y H, Zhou Z W. Numerical simulation of Rayleigh breakup in low-velocity liquid jet. Journal of Shanghai University, 2008; 14(2): 161-167. (in Chinese)

[22] Pan Y, Suga K. Capturing the pinch-off of the liquid jets by the level set method. Journal of Fluids Engineering, 2003; 125: 922-930.

[23] Tian X S, Zhou H, Liu H F. Three-dimensional large eddy simulation of round liquid jet primary breakup in coaxial gas flow using the VOF method. Fuel Processing Technology, 2015; 131: 396-402.

[24] Ménard T, Tanguy S, Berlemont A. Coupling level set/VOF/ghost fluid methods: Validation and application to $3 \mathrm{D}$ simulation of the primary break-up of a liquid jet. International Journal of Multiphase Flow, 2007; 33: $510-524$

[25] Shinjo J, Umemura A. Simulation of liquid jet primary breakup: Dynamics of ligament and droplet formation. International Journal of Multiphase Flow, 2010; 36: 513-532.

[26] Thakre S, Manickam L, Ma W M. A numerical simulation of jet breakup in melt coolant interactions. Annals of Nuclear Energy, 2015; 80: 467-475.

[27] Shi S X, Du Q, Qin J R. Temporal mode and spatial mode in the study of liquid jet breakup. Transactions of the CSICE, 1999; 17(3): 205-210.

[28] Blaisot J B, Adeline, S. Determination of the growth rate of instability of low velocity free falling jets. Experiments in Fluids, 2000; 29: 247-256.

[29] GB/T 19795.2-2005, Rotating Sprinkler. 2005; pp.1-4.

[30] Bezdek J C, Solomon K H. Upper limit log normal distribution for drop size data. Journal of Irrigation and Drainage Engineering, 1983; 109(1): 72-89.

[31] Chang W H, Chen L F, Wang L X. On spray range of water flow in sprinkler irrigation. Transactions of the CSAM, 1991; 22(4): 46-52. (in Chinese)

[32] Tang Y, Zhu X Y, Zheng Y. Simulated experiment of the turbo-type whirling sprinkler for achieving variable rate irrigation. China Rural Water and Hydropower, 2009; 8: 4-7.

[33] Feng C D. Calculation of spray range. Drainage \& Irrigation Machinery, 1986; 5(4): 35-38. (in Chinese)

[34] Gan Z M, Yang S H. The formula and experimental research on the range of the whirl sprinkler. Transactions of the CSAM, 1998; 29(4): 145-149. (in Chinese) 